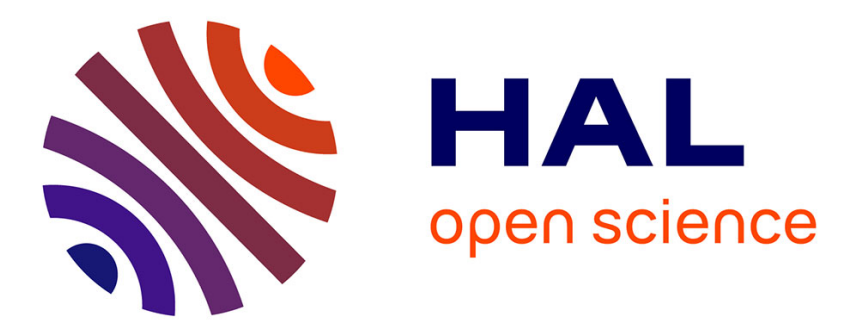

\title{
Uniting control laws: On obstacle avoidance and global stabilization of underactuated linear systems
}

Philipp Braun, Christopher M Kellett, Luca Zaccarian

\section{To cite this version:}

Philipp Braun, Christopher M Kellett, Luca Zaccarian. Uniting control laws: On obstacle avoidance and global stabilization of underactuated linear systems. 58th IEEE Conference on Decision and Control, Dec 2019, Nice, France. hal-02072184

\section{HAL Id: hal-02072184 \\ https://hal.science/hal-02072184}

Submitted on 19 Mar 2019

HAL is a multi-disciplinary open access archive for the deposit and dissemination of scientific research documents, whether they are published or not. The documents may come from teaching and research institutions in France or abroad, or from public or private research centers.
L'archive ouverte pluridisciplinaire HAL, est destinée au dépôt et à la diffusion de documents scientifiques de niveau recherche, publiés ou non, émanant des établissements d'enseignement et de recherche français ou étrangers, des laboratoires publics ou privés. 


\title{
Uniting control laws: On obstacle avoidance and global stabilization of underactuated linear systems
}

\author{
Philipp Braun ${ }^{1}$, Christopher M. Kellett ${ }^{1}$ and Luca Zaccarian $^{2}$
}

\begin{abstract}
An important problem in safety-critical applications is that of simultaneous stabilization (of the origin) and obstacle avoidance. When obstacles are defined by bounded sets of the state space, state feedback controllers are necessarily discontinuous and can be naturally formulated in the framework of hybrid systems. In previous work, we developed such hybrid controllers for linear systems when a point obstacle is prohibited from being an induced equilibrium of a single-input system or for multi-input systems when the input matrix has full row rank. In this paper, we explore a design for simultaneous stabilization of the origin and avoidance of an obstacle for single-input systems when the point obstacle is an induced equilibrium. The presented Lyapunov-based feedback control design provably works for planar systems and may work for systems of even degree.
\end{abstract}

\section{INTRODUCTION}

Global asymptotic stability properties of linear systems are well understood. For example, pole placement or optimal control discussed in almost every introductory textbook on mathematical control theory can be used to design linear feedback laws stabilizing equilibria or invariant sets. Aside from stabilizing controllers investigating the convergence towards a target set, dual concepts have been proposed to ensure that a controller avoids certain unsafe areas or obstacles in the state space. For example, barrier functions have been introduced and defined in [15], as a certificate to ensure that predefined regions in the state space are avoided.

While these independent concepts, i.e., stability and obstacle avoidance, are well understood individually, combining both concepts to define control laws that simultaneously guarantee global asymptotic stability of a target set and obstacle avoidance are less studied and the available results are far from being complete. This is especially true in the case of bounded obstacles, where discontinuous feedback laws need to be considered due to topological obstructions, as argued in [9, Ch. 4], for example. The topological obstructions caused by bounded obstacles are particularly challenging in controller design for underactuated systems where it is not possible to move in every direction and the natural drift of the system needs to be respected.

* P. Braun and C. M. Kellett are supported by the Australian Research Council (Grant number: ARC-DP160102138). L. Zaccarian is supported in part by ANR via project HANDY, number ANR-18-CE40-0010.

1 P. Braun and C. M. Kellett are with the School of Electrical Engineering and Computing at the University of Newcastle, Callaghan, New South Wales 2308, Australia, \{chris.kellett, philipp.braun $\}$ anewcastle.edu.au.

${ }^{2} \mathrm{~L}$. Zaccarian is with the Laboratoire d'Analyse et d'Architecture des Systèmes-Centre Nationnal de la Recherche Scientifique, Université de Toulouse, CNRS, UPS, 31400 Toulouse, France, and also with the Dipartimento di Ingegneria Industriale, University of Trento, 38122 Trento, Italy, luca.zaccarian@laas.fr.
The combined control problem, i.e., obstacle avoidance plus target set stabilization, is also the motivation for artificial potential fields originating in the works [7], [8]. However, artificial potential fields are in general only discussed for fully actuated dynamical systems and avoidance and stability results only hold for domains in the state space excluding sets of measure zero.

In this paper we study the simplest setting of combined robust global stabilization and obstacle avoidance of a bounded set for underactuated systems. More specifically, we consider an $n$-dimensional linear system

$$
\dot{x}=A x+B u, \quad x_{0}=x(0) \in \mathbb{R}^{n}
$$

where we want to globally asymptotically stabilize the origin $x=0$, while avoiding an obstacle described through a single point $x_{a} \in \mathbb{R}^{n} \backslash\{0\}$ for all initial values $x(0) \in \mathbb{R}^{n} \backslash\left\{x_{a}\right\}$. A rigorous understanding of this setting is necessary before more complicated dynamics, invariant sets, and obstacles can be studied.

Under the assumption that $B \in \mathbb{R}^{n \times 1}$ (i.e., the input $u$ is one dimensional) and the pair $(A, B)$ is stabilizable, a solution of the combined control problem in the form of a hybrid switching control law is provided in [4] for points $x_{a}$ satisfying

$$
x_{a} \notin\left\{y \in \mathbb{R}^{n}: \exists \nu \in \mathbb{R}, A y+B \nu=0\right\} .
$$

In words, this means that the obstacle to be avoided cannot be an induced equilibrium of (1); that is, there cannot be a constant control input that maintains the system state at $x_{a}$. In [3] it is additionally argued that in the case $B \in \mathbb{R}^{n \times m}$, $m>1$ (and $B$ has rank $m$ ), the hybrid controller design can be used for all $x_{a} \in \mathbb{R}^{n} \backslash\{0\}$. In this paper we thus address the problem $B \in \mathbb{R}^{n \times 1}$ and

$$
x_{a} \in\left\{y \in \mathbb{R}^{n}: \exists \nu \in \mathbb{R}, A y+B \nu=0\right\}
$$

which means that $x_{a}$ is an induced equilibrium.

We provide here a fully constructive solution for planar systems $n=2$. Additionally, we conjecture that the controller leads to the same results if the dimension $n \in \mathbb{N}$ is even. As it turns out, it appears that for odd dimensions $n \in$ $\mathbb{N}$, if possible, a fundamentally different approach to solve the combined control problem is necessary. Specifically, the controller proposed in this paper does not solve the combined control problem for $n \in \mathbb{N}$ odd.

Our work is highly influenced by the controller design in [12] and [13], uniting local and global control laws in a hybrid setting. In the same way as in our earlier work [3] 
and [4], we embed our controller design in the framework of hybrid dynamical systems and follow the descriptions and notations used in the monograph [6].

The paper is structured as follows. In Section II, the setting is introduced and the problem is formulated. In Section III, stabilizing and destabilizing controllers are defined, solving the individual avoidance and stabilization problems. The difficulties in combining these control laws to simultaneously guarantee avoidance and stability for underactuated systems are highlighted in two examples in Section IV. The main results of the paper are derived in Section $\mathrm{V}$, in terms of a hybrid controller, solving the combined control problem for two dimensional linear systems. Numerical examples are given in Section VI before the paper concludes with final comments in Section VII.

\section{Setting \& PROBLEM FORMUlation}

Consider linear system (1) with state $x \in \mathbb{R}^{n}$, one dimensional input $u \in \mathbb{R}$ and matrices $A \in \mathbb{R}^{n \times n}, B \in \mathbb{R}^{n}$, and assume that the pair $(A, B)$ is controllable. For a given feedback law $u: \mathbb{R}^{n} \rightarrow \mathbb{R}$, the solution of the linear system is denoted by $x\left(\cdot ; x_{0}, u\right): \mathbb{R}_{\geq 0} \rightarrow \mathbb{R}^{n}$. As motivated in the introduction, we consider the problem discussed in [3] and [4] but we make different assumptions on the linear systems and on the unsafe point $x_{a} \in \mathbb{R}^{n} \backslash\{0\}$. In particular, we will address the following problem:

Problem 1: (Semiglobal $x_{a}$-avoidance augmentation with GAS) Given an "unsafe" point $x_{a} \in \mathbb{R}^{n}$ that must be avoided by the controller, and a stabilizing state feedback $u_{s}(x)=$ $K_{s} x$, design a feedback selection of $u(x)$ that guarantees

(i) (GAS) uniform global asymptotic stability of the origin;

(ii) (semiglobal preservation) the feedback matches the original stabilizer $u(x)=u_{s}(x)=K_{\mathrm{s}} x$ except for a neighborhood around $x_{a}$; and

(iii) (semiglobal $x_{a}$-avoidance) all solutions starting a certain distance away from $x_{a}$ never enter a suitable "safety" neighborhood of $x_{a}$.

Assumption 1: Basic assumptions:

(a) The pair $(A, B)$ is controllable.

(b) The unsafe point $x_{a} \in \mathbb{R}^{n} \backslash\{0\}$ is in the set of induced equilibria

$$
x_{\mathrm{a}} \in \mathcal{E}:=\left\{y \in \mathbb{R}^{n}: \exists \nu \in \mathbb{R}, 0=A y+B \nu\right\} .
$$

Note that in [3], [4] the case $x_{a} \notin \mathcal{E}$ is discussed and solved. Additionally, for $x_{a} \notin \mathcal{E}$, stabilizability of the pair $(A, B)$ is sufficient. As it turns out, to solve Problem 1 for $x_{a} \in \mathcal{E}$, controllability is generally necessary (see Section IV-A).

To simplify calculations and notations with respect to the subspace of equilibria $\mathcal{E}$ in the following, we assume without loss of generality that the matrix $A$ is invertible and thus $\mathcal{E}$ defined in (2) is given by

$$
\mathcal{E}=\operatorname{span}\left(A^{-1} B\right),
$$

where $\operatorname{span}(y)=\left\{x \in \mathbb{R}^{n}: x=\lambda y, \lambda \in \mathbb{R}\right\}$ denotes the one dimensional space generated by a vector $y \in \mathbb{R}^{n} \backslash\{0\}$.
If $A$ is not invertible, we can consider the system $\dot{x}=$ $A x+B K x+B v$ with $v=u-K x$ and $A+B K$ invertible instead, which is always possible due to the controllability assumption on $(A, B)$. Note that this transformation does not change $\mathcal{E}$.

In the following we will provide a comprehensive and explicit solution for Problem 1 under Assumption 1 for two dimensional systems (i.e., $n=2$ ). For $n \in \mathbb{N}$ even and $n \geq 4$, we are currently unable to verify global asymptotic stability of the closed loop using the approach presented below, i.e., Problem 1(i) is not provably solved by our approach. Surprisingly, if at all possible, for $n \in \mathbb{N}$ odd, our approach is not applicable and a fundamentally different solution seems to be necessary.

\section{Stabilizing AND DESTABILIZING CONTROLlER DESIGN}

\section{A. Construction of stabilizing and destabilizing controllers}

The control law providing a solution to Problem 1 will be composed of three independent control laws:

1. a stabilizing controller $u_{s}: \mathbb{R}^{n} \rightarrow \mathbb{R}$ globally asymptotically stabilizing the origin $x=0$,

2. a stabilizing controller $u_{s a}: \mathbb{R}^{n} \rightarrow \mathbb{R}$ globally asymptotically stabilizing the point $x_{a} \in \mathcal{E}$, and

3. a destabilizing controller $u_{d}: \mathbb{R}^{n} \rightarrow \mathbb{R}$ globally completely destabilizing the point $x_{a} \in \mathcal{E}$.

Here, in contrast to instability, complete instability means that for every initial condition $x_{0} \in \mathbb{R} \backslash\left\{x_{a}\right\}$ the corresponding solution $x\left(\cdot ; x_{0}, u_{d}\right)$ drifts away from the point $x_{a}$ (e.g., instability as it is used and defined in [11] or [2]).

Since $u_{s}$ is a special case of $u_{s a}$ we start with the construction of $u_{s a}$. To this end, let $\eta_{x_{a}} \in \mathbb{R}$ denote the input such that

$$
A x_{\mathrm{a}}+B \eta_{x_{a}}=0
$$

is satisfied. We consider the coordinate transformation $y=$ $x-x_{\mathrm{a}}$ and $v=u-\eta_{x_{a}}$ leading to the dynamics

$$
\dot{y}=\overbrace{x-x_{\mathrm{a}}}^{ن}=A\left(x-x_{\mathrm{a}}\right)+B\left(u-\eta_{x_{a}}\right)=A y+B v .
$$

Since $(A, B)$ is controllable, we can use pole placement (or linear quadratic optimal control) to construct a feedback gain matrix $K_{s a} \in \mathbb{R}^{1 \times n}$ such that $A_{s a}=A+B K_{s a}$ is Hurwitz and the equilibrium $y=0$ in (5) using the feedback $v=K_{s a} y$ is globally asymptotically stable. Thus $u_{s a}$ can be defined as

$$
u_{s a}(x)=K_{s a} x-K_{s a} x_{a}+\eta_{x_{a}},
$$

which globally asymptotically stabilizes the induced equilibrium $x_{a}$. Additionally, for the feedback control that stabilizes the origin, this implies that $u_{s}$ can be defined as

$$
u_{s}(x)=K_{s} x
$$

where $A_{s}=A+B K_{s}$ is Hurwitz for $K_{s} \in \mathbb{R}^{1 \times n}$. The feedback gains $K_{s}$ and $K_{s a}$ can be defined to be equal, but can also be defined independently. 
To construct a controller $u_{d}$ that destabilizes $x_{a}$, we consider the time reversal system of (5), i.e.,

$$
\dot{z}=-A z-B v
$$

with $z(t)=y(-t)$. Since the pair $(-A,-B)$ is controllable, $K_{d} \in \mathbb{R}^{1 \times n}$ can again be defined in such a way that $-\left(A+B K_{d}\right)$ is Hurwitz. Using the feedback $v(y)=K_{d} y$ implies that $y=0$ is completely unstable for the closed-loop dynamics (5). Thus, the destabilizing control law $u_{d}$ can be defined as

$$
u_{d}(x)=K_{d} x-K_{d} x_{a}+\eta_{x_{a}}
$$

We use the notation $A_{d}=A+B K_{d}$ to denote the closed loop matrix of the destabilizing controller.

\section{B. Lyapunov and Chetaev functions}

To obtain stability certificates we use Lyapunov and Chetaev functions. Here, we follow the notation in [2] using Chetaev functions to refer to instability, in contrast to Lyapunov functions to describe stability properties. Since $A_{s}, A_{s a}$, and $-A_{d}$ are Hurwitz, the Lyapunov equations

$$
\begin{aligned}
A_{s}^{T} P_{s}+P_{s} A_{s} & =-Q_{s} \\
A_{s a}^{T} P_{s a}+P_{s a} A_{s a} & =-Q_{s a} \\
-A_{d}^{T} P_{d}-P_{d} A_{d} & =-Q_{d}
\end{aligned}
$$

have unique positive definite solutions $P_{s}, P_{s a}, P_{d} \in \mathbb{R}^{n \times n}$ for positive definite matrices $Q_{s}, Q_{s a}, Q_{d} \in \mathbb{R}^{n \times n}$. Moreover, the Lyapunov functions $V_{s}, V_{s a}: \mathbb{R}^{n} \rightarrow \mathbb{R}_{\geq 0}$,

$$
\begin{aligned}
V_{s}(x) & =x^{T} P_{s} x, \\
V_{s a}(x) & =\left(x-x_{a}\right)^{T} P_{s a}\left(x-x_{a}\right)
\end{aligned}
$$

satisfy the decrease conditions

$$
\begin{aligned}
\left\langle\nabla V_{s}(x), A x+B u_{s}(x)\right\rangle<0, & & \forall x \in \mathbb{R}^{n} \backslash\{0\} \\
\left\langle\nabla V_{s a}(x), A x+B u_{s a}(x)\right\rangle<0, & & \forall x \in \mathbb{R}^{n} \backslash\left\{x_{a}\right\}
\end{aligned}
$$

while the Chetaev function $C_{d}: \mathbb{R}^{n} \rightarrow \mathbb{R}_{\geq 0}$,

$$
C_{d}(x)=\left(x-x_{a}\right)^{T} P_{d}\left(x-x_{a}\right)
$$

satisfies the increase condition

$$
\left\langle\nabla C_{d}(x), A x+B u_{d}(x)\right\rangle>0, \quad \forall x \in \mathbb{R}^{n} \backslash\left\{x_{a}\right\} .
$$

\section{Motivating EXAMPLES}

Before we combine the control laws (6), (7), and (9) in a hybrid control framework, we discuss here two examples of obstacle avoidance and target set stabilization for underactuated systems highlighting the difficulties in the construction of robust control laws.

\section{A. Necessity of the controllability assumption}

We consider the two dimensional stabilizable linear system

$$
\left[\begin{array}{l}
\dot{x}_{1} \\
\dot{x}_{2}
\end{array}\right]=\left[\begin{array}{rr}
1 & 0 \\
0 & -1
\end{array}\right]\left[\begin{array}{l}
x_{1} \\
x_{2}
\end{array}\right]+\left[\begin{array}{l}
1 \\
0
\end{array}\right] u
$$

to demonstrate that the controllability assumption is essential for avoiding a point $x_{a} \in \mathcal{E}$. Note that this is different from the case $x_{a} \notin \mathcal{E}$ as demonstrated in [4], where stabilizability is sufficient. It is easy to verify that the linear system (14) is stabilizable but not controllable. The subspace of induced equilibria (2) is given by $\mathcal{E}=\operatorname{span}\left((1,0)^{T}\right)$.

Since a solution $x\left(\cdot ; x_{0}, u\right)$ with initial value $x_{0} \in \mathcal{E}$ satisfies $x\left(t ; x_{0}, u\right) \in \mathcal{E}$ for all $t \in \mathbb{R}_{\geq 0}$ regardless of $u$, Problem 1 cannot be solved. In particular, it is possible to avoid $x_{a}$ but simultaneous global asymptotic stability cannot be guaranteed for all initial values $x_{0} \in \mathbb{R}^{n} \backslash\left\{x_{a}\right\}$ because all solutions $x\left(\cdot ; x_{0}, u\right)$ starting at $x_{0}=\ell x_{a}$ with $\ell>1$ must hit $x_{a}$ if they approach the origin.

With respect to the construction of the destabilizing controller (9), observe that this construction is not possible if $(A, B)$ is stabilizable but not controllable. In particular if $(A, B)$ is stabilizable but not controllable, then $(-A,-B)$ is not stabilizable (and not controllable). Thus a stabilizing control law for (8) (which completely destabilizes the induced equilibria $x_{a}$ of the linear system (5)) does not exist.

\section{B. Implications of the drift term}

As a second example we consider the dynamics of the controllable linear system

$$
\left[\begin{array}{l}
\dot{x}_{1} \\
\dot{x}_{2}
\end{array}\right]=\left[\begin{array}{rr}
0 & -1 \\
1 & 0
\end{array}\right]\left[\begin{array}{l}
x_{1} \\
x_{2}
\end{array}\right]+\left[\begin{array}{l}
1 \\
0
\end{array}\right] u .
$$

The quiver plot of the uncontrolled dynamics is visualized in Figure 1. The red arrows pointing to the right show the direction $B$ and the blue arrows visualize the drift term $A x$.

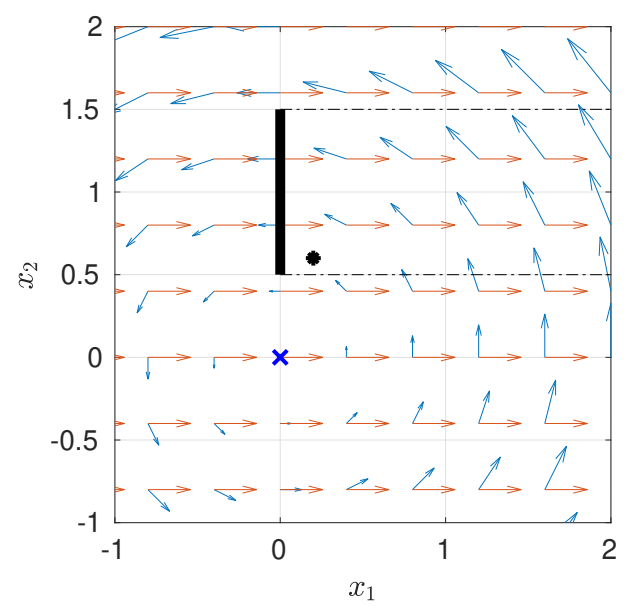

Fig. 1. Quiver plot of the dynamical system (15). The blue arrows indicate the direction of the drift term $A x$ and the red arrows pointing to the right indicate the direction $B$. The thick solid block indicates a possible obstacle in $\mathcal{E}$. Initial values with $x_{1}(0)>0$ arbitrarily small and $x_{2}(0)=0.5$ can only increase $x_{2}$ to avoid the obstacle. Due to the limitations in the input, $x_{2}$ cannot be decreased immediately. 
The thick solid block $[0.5,1.5] \times\{0\}$, represents a possible obstacle in the one dimensional subspace $\mathcal{E}=\operatorname{span}\left((0,1)^{T}\right)$.

Because the system is underactuated, the possible directions $A x+B u$ are limited. This implies that the drift term must be actively used to avoid the obstacle and the drift term cannot simply be equalized by the input $u$ before augmenting the controller with an additional stabilizer and avoidance controller.

Consider for example the initial value $x(0)=(0.2,0.6)^{T}$. Due to the orientation of the vector $B$, the input $u$ can only influence the $x_{1}$-component of a closed-loop solution. Due to the drift, it holds that $\dot{x}_{2}>0$ whenever $x_{1}>0$. Thus $[0.5,1.5] \times\{0\}$ can only be avoided by increasing $x_{2}$ resulting in a possibly large detour before the origin is reached. Additionally, $u$ can only be used to stall time, keeping $x\left(t ; x_{0}, u\right)$ away from the obstacle, while $A x$ is actually responsible for the avoidance.

This example highlights the difficulties in the controller design for underactuated systems.

\section{A HYBRID CONTROLLER DESIGN}

In this section we propose a hybrid controller switching between stabilizing and avoidance control laws. The section is divided in three parts. In Section V-A, we introduce sets that will be used later to define the jump set and flow set of a hybrid system. In Section V-B we define and analyze an avoidance controller based on the definitions of $u_{s a}$ and $u_{d}$ in (6) and (9). Finally, in Section V-C the avoidance controller is combined with the stabilizing controller $u_{s}$ defined in (7), to guarantee avoidance and stability of the closed loop, at least in the two dimensional setting.

\section{A. Key ingredients for defining jump and flow sets}

To derive avoidance and stability properties, we will make use of the fact that sublevel sets of Lyapunov functions, and superlevel sets of Chetaev functions, define forward invariant sets. For a given function $H: \mathbb{R}^{n} \rightarrow \mathbb{R}_{\geq 0}$ and a positive constant $r>0$ we define the set

$$
L_{\backsim}^{r}(H)=\left\{x \in \mathbb{R}^{n}: H(x) \backsim r\right\}
$$

where $\backsim$ denotes a relation $\backsim \in\{<, \leq,=, \geq,>\}$.

Of particular interest in this paper are the level sets of the functions (11)-(13) visualized in Figure 2. If $x_{0} \in L_{\leq}^{\sigma}\left(V_{s}\right)$ for a given $\sigma>0$ and the control law $u_{s}$ defined in (7) is used as an input, then the closed loop satisfies $x\left(t ; x_{0}, u_{s}\right) \rightarrow 0$ for $t \rightarrow \infty$ and $x\left(t ; x_{0}, u_{s}\right) \in L_{<}^{\sigma}\left(V_{s}\right)$ for all $t \in \mathbb{R}_{\geq 0}$. Similarly, for $x_{0} \in L_{\leq}^{\alpha}\left(V_{s a}\right)$ and $\alpha>0$, the solution $x\left(\cdot ; x_{0}, u_{s a}\right)$ satisfies $x\left(t ; x_{0}, u_{s a}\right) \in L_{<}^{\alpha}\left(V_{s a}\right)$ for all $t \in \mathbb{R}_{\geq 0}$. The destabilizing controller on the other hand guarantees that for any $\delta>0, x\left(t ; x_{0}, u_{d}\right) \notin L_{<}^{\delta}\left(C_{d}\right)$ for all $t \in \mathbb{R}_{\geq 0}$ as long as $x_{0} \in L_{\geq}^{\delta}\left(C_{d}\right)$.

If the constants $\alpha, \delta \in \mathbb{R}_{>0}$ are selected such that

$$
L_{\leq}^{\delta}\left(C_{d}\right) \subset L_{\leq}^{\alpha}\left(V_{s a}\right)
$$

(as visualized in Figure 2, for example), then $u_{d}$ and $u_{s a}$, can be used as an input to ensure that a solution of the dynamical

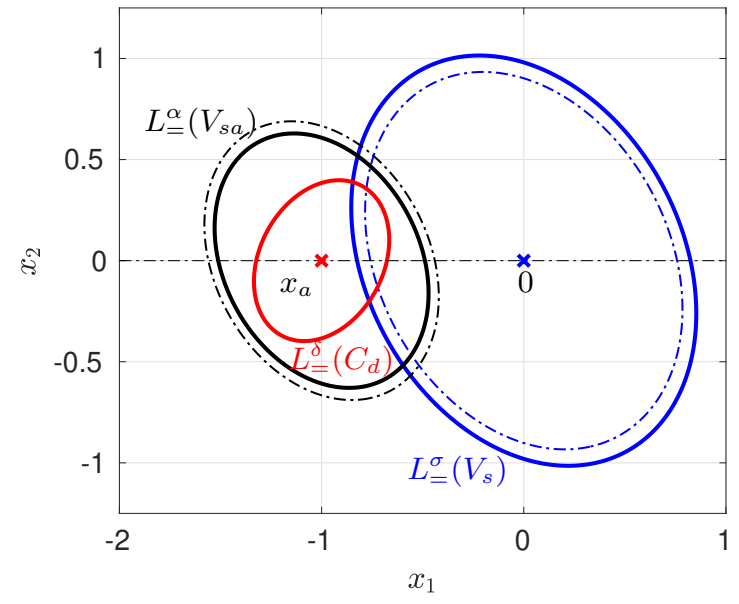

Fig. 2. Level sets of the Lyapunov functions $V_{s}, V_{s a}$, and the Chetaev function $C_{d}$ defined in (11), (12), and (13) for given constants $\sigma, \alpha, \delta \in$ $\mathbb{R}_{>0}$. The dashed lines show a slightly enlarged and a slightly smaller level set $\alpha+h$ and $\sigma-h$ based on a positive hysteresis parameter $h \in \mathbb{R}_{>0}$.

system (1) starting in the donut

$$
x_{0} \in \mathcal{S}:=L_{\leq}^{\alpha}\left(V_{s a}\right) \backslash L_{<}^{\delta}\left(C_{d}\right)
$$

stays in the donut for all $t \in \mathbb{R}_{\geq 0}$. This can simply be achieved by demanding that $u(x)=u_{s a}(x)$ for all $x \in$ $L_{=}^{\alpha}\left(V_{s a}\right)$ and $u(x)=u_{d}(x)$ for all $L_{=}^{\delta}\left(C_{d}\right)$, which is made more precise in the next section.

The dashed lines in Figure 2 show slightly larger and smaller level sets $L_{<}^{\alpha+h}\left(V_{s a}\right)$ and $L_{<}^{\sigma-h}\left(V_{s}\right)$, respectively, for a positive hysteresis parameter $h \in \mathbb{R}_{>0}$. The parameter $h$ will be used in the following sections to ensure robustness properties while switching between control laws.

\section{B. Analysis of the avoidance controller}

In this section we concentrate on a combination of the control laws $u_{s a}$ defined in (6) and the control law $u_{d}$ defined in (9). Additionally we assume that $\alpha, \delta \in \mathbb{R}_{>0}$ are selected such that (17) is satisfied.

Lemma 1: Let $\mu: \mathbb{R}^{n} \rightarrow[0,1]$ be defined as

$$
\mu(x)= \begin{cases}1, & \text { if } x \in L_{\geq}^{\alpha}\left(V_{s a}\right) \\ \frac{1-\delta^{-1} C_{d}(x)}{\alpha^{-1} V_{s a}(x)-\delta^{-1} C_{d}(x)}, & \text { if } x \in \mathcal{S} \\ 0, & \text { if } x \in L_{\leq}^{\delta}\left(C_{d}\right)\end{cases}
$$

with $\mathcal{S}$ defined in (18), and let $u_{a}: \mathbb{R}^{n} \rightarrow \mathbb{R}$ be defined as

$$
u_{a}(x)=\mu(x) u_{s a}(x)+(1-\mu(x)) u_{d}(x) .
$$

Then, for any initial value $x_{0} \in \mathbb{R}^{n} \backslash\left\{x_{a}\right\}$ the solution $x\left(\cdot ; x_{0}, u_{a}\right)$ of the linear system (1) using the control law (19) is such that:

(a) there exists $T \in \mathbb{R}_{\geq 0}$ such that $x\left(T ; x_{0}, u_{a}\right) \in \mathcal{S}$, and, (b) $x\left(t ; x_{0}, u_{a}\right) \in \mathcal{S}$ for all $t \geq T$.

In other words, the controller $u_{a}$ renders the set $\mathcal{S}$ attractive and forward invariant. 
Proof: First note that $\mu$ is defined based on the condition

$$
1=\mu(x) \frac{1}{\alpha} V_{s a}(x)+(1-\mu(x)) \frac{1}{\delta} C_{d}(x)
$$

for $x \in \mathcal{S}$. Condition (17) ensures that $\mu$ is well defined, continuous, and $\mu(x) \in[0,1]$ for all $x \in \mathbb{R}^{n}$. With this selection of $\mu$, the definition of $u_{a}(x)$ in (19) ensures that $u_{a}(x)=u_{d}(x)$ if $x \in L_{\leq}^{\delta}\left(C_{d}\right), u_{a}(x)=u_{s a}(x)$ if $x \in L_{\geq}^{\alpha}\left(V_{s a}\right)$, and $u_{a}(x)$ is a convex combination of $u_{d}(x)$ and $u_{s a}(x)$ if $x \in \mathcal{S}$. The stabilizing controller $u_{s a}$ and the destabilizing controller $u_{d}$ guarantee that every solution $x\left(\cdot ; x_{0}, u_{a}\right), x_{0} \neq x_{a}$, reaches the set $\mathcal{S}$ in finite time $T\left(x_{0}\right) \in \mathbb{R}_{\geq 0}$. The selection of $u_{a}=u_{s a}$ and $u_{a}=u_{d}$ on the outer and inner boundary of $\mathcal{S}$ additionally ensures that solutions cannot escape $\mathcal{S}$ for $t \geq T\left(x_{0}\right)$.

In the remainder of this section we characterize the behavior of solutions in $\mathcal{S}$. In particular, we analyze if there exist induced equilibria $x \in \mathbb{R}^{n}, x \neq x_{a}$, satisfying

$$
0=A x+B u_{a}(x),
$$

with $u_{a}$ in (19). Remember that induced equilibria can only arise in the one-dimensional subspace $\mathcal{E}$.

Lemma 2: Consider linear system (1), let $x_{a} \in \mathcal{E}$ and let $u_{s a}$ and $u_{d}$ be defined as in (6) and (9). Then, for all $x \in \mathcal{E}$ it holds that

$$
A x+B u_{s a}(x)=\rho\left(A x+B u_{d}(x)\right),
$$

where $\rho \in \mathbb{R}$ is defined as

$$
\rho=\frac{1+K_{s a} A^{-1} B}{1+K_{d} A^{-1} B} .
$$

Additionally, $\rho$ satisfies

$$
\operatorname{sign}(\rho)=(-1)^{n},
$$

where $n \in \mathbb{N}$ is the dimension of $x$.

Proof: Let $x \in \mathcal{E}$ be arbitrary. Since $x_{a} \in \mathcal{E}$ by definition, also vector $x-x_{a}$ belongs to the subspace $\mathcal{E}=\operatorname{span}\left(-A^{-1} B\right)$. Thus, there exists $\kappa \in \mathbb{R}$ such that $x-x_{a}=-A^{-1} B \kappa$. Then the dynamics of linear system (1) using controller $u_{s a}$ correspond to

$$
\begin{aligned}
\dot{x} & =A x+B u_{s a}(x) \\
& =A x+B K_{s a}\left(x-x_{a}\right)+B \eta_{x a} \\
& =A\left(x-x_{a}\right)+B K_{s a}\left(x-x_{a}\right) \\
& =-B\left(1+K_{s a} A^{-1} B\right) \kappa .
\end{aligned}
$$

Similarly, for the destabilizing controller in (9) we obtain

$$
\dot{x}=A x+B u_{d}(x)=-B\left(1+K_{d} A^{-1} B\right) \kappa
$$

which proves identity (20).

To prove identity (21), we denote the eigenvalues of $A+B K_{s}$ and $A+B K_{d}$ by $\lambda_{1}^{s}, \ldots, \lambda_{n}^{s}$ and $\lambda_{1}^{d}, \ldots, \lambda_{n}^{d}$, respectively. Since $A+B K_{s}$ and $-\left(A+B K_{d}\right)$ are Hurwitz, the real parts of the eigenvalues satisfy $\operatorname{Re}\left(\lambda_{i}^{s}\right)<0$ and $\operatorname{Re}\left(\lambda_{i}^{d}\right)>0$ for all $i=1, \ldots, n$. (Additionally note that a pair of complex conjugate eigenvalues satisfies $(a+i b)(a-$ $i b)=a^{2}+b^{2}>0$ independent of the sign of $a, b \in \mathbb{R} \backslash\{0\}$.)
With these notations it holds that

$$
\begin{aligned}
\prod_{i=1}^{n} \lambda_{i}^{s} & =\operatorname{det}\left(A+B K_{s}\right) \\
& =\operatorname{det}(A) \operatorname{det}\left(1+K_{s} A^{-1} B\right) \\
& =\operatorname{det}(A)\left(1+K_{s} A^{-1} B\right)
\end{aligned}
$$

where the second equality follows from Sylvester's determinant theorem [14], [1]. In the same way, the equality

$$
\prod_{i=1}^{n} \lambda_{i}^{d}=\operatorname{det}(A)\left(1+K_{d} A^{-1} B\right)
$$

is obtained. Since

$$
\operatorname{sign}\left(\prod_{i=1}^{n} \lambda_{i}^{s}\right)=(-1)^{n} \quad \text { and } \quad \operatorname{sign}\left(\prod_{i=1}^{n} \lambda_{i}^{d}\right)=1,
$$

the identity (21) follows. (Recall that in (3) we showed that $A$ invertible can be assumed without loss of generality, which ensures the existence of $A^{-1}$ and $\operatorname{det}(A) \neq 0$.)

Lemma 2 allows us to characterize the equilibria of the nonlinear dynamical system

$$
\dot{x}=A x+B u_{a}(x)
$$

(which is nonlinear due to the control law $u_{a}$ defined in (19)), where the number of equilibria apparently is independent of the control laws $u_{s a}$ and $u_{d}$ and only depends on the dimension $n$.

The relevance of Lemma 2 is highlighted next.

Corollary 1: Consider the nonlinear system (22) of dimension $n \in \mathbb{N}, n \geq 2$, where $u_{a}$ is defined in (19).

(a) If $n$ is even, then $x_{a} \in \mathcal{E}$ is the only equilibrium of (22).

(b) If $n$ is odd, then (22) has two equilibria in $\mathcal{E} \cap \mathcal{S}$ as well as $x_{a} \in \mathcal{E}$ as an equilibrium.

Proof: The result follows immediately from Lemma 2. As already pointed out, equilibria can only occur on the subspace $\mathcal{E}$. Additionally, by construction, $\dot{x}=A x+$ $B u_{s a}(x)$ and $\dot{x}=A x+B u_{d}(x)$ have $x_{a}$ as their only equilibrium. Since $u_{d}=u_{a}$ in a neighborhood of $x_{a}$, then $x_{a}$ is an equilibrium of (22). From Lemma 2 we know that if $n$ is even, then $A x+B u_{s a}(x)$ and $A x+B u_{d}(x)$ are linearly dependent and point in the same direction for all $x \in \mathcal{E} \backslash\left\{x_{a}\right\}$. Thus a convex combination (19) cannot lead to an equilibrium which proves item (a). If $n$ is odd, then $A x+B u_{s a}(x)$ and $A x+B u_{d}(x)$ are linearly dependent and point in opposite directions. Thus the convex combination (19) introduces an equilibrium. Due to symmetry of the Lyapunov function $V_{s a}$ and the Chetaev function $C_{d}$ with respect to the point $x_{a}$ and the subspace $\mathcal{E}$, two equilibria are introduced, proving (b).

Control law $u_{a}$ in (19) guarantees global avoidance of the point $x_{a}$ and boundedness of solutions of the linear system (1). In the next section we embed the control law $u_{a}$ in a hybrid control framework and combine it with the control law $u_{s}$ defined in (7), to discuss additional robust global stability properties for $n=2$.

We conclude this section with the following observation. 
Lemma 3: Consider the nonlinear dynamical system (22) in the planar case $n=2$. Then the set $\mathcal{S}$ contains a periodic orbit centered around $x_{a}$.

Proof: The result follows immediately from the Poincaré-Bendixson Theorem (see [10, Thm. 6.12], for example) combined with Lemma 1, Lemma 2, and Corollary 1.

\section{The hybrid avoidance controller stabilizing the origin}

In this section we combine the control law $u_{a}$ defined in (19) with the stabilizing control law $u_{s}$ defined in (7). We assume that the parameters $\sigma, \alpha, \delta \in \mathbb{R}_{>0}$ and hysteresis parameter $h \in \mathbb{R}_{>0}$ are selected such that the following properties are satisfied:

$$
\begin{aligned}
& L_{\leq}^{\delta}\left(C_{d}\right) \subset L_{\leq}^{\alpha}\left(V_{s a}\right), \\
& L_{<}^{\delta}\left(C_{d}\right) \cap L_{<}^{\sigma-h}\left(V_{s}\right) \neq \emptyset, \\
& x_{a} \notin L_{\leq}^{\sigma}\left(V_{s}\right) .
\end{aligned}
$$

Note that the selection of $\sigma, \alpha, \delta, h \in \mathbb{R}_{>0}$ satisfying these properties is always possible, for every $V_{s}, V_{s a}$, and $C_{d}$. (In the setting visualized in Figure 2, conditions (23) are satisfied.)

To obtain a hybrid system with a switching control law we augment the state $x$ and define $\xi=\left(x^{T}, q\right)^{T}$ where $q \in$ $\{0,1\}$ is responsible for the controller selection. In particular we define the feedback selection

$$
\gamma(\xi)= \begin{cases}u_{s}(x), & \text { if } q=0 \\ u_{a}(x), & \text { if } q=1\end{cases}
$$

and the flow map

$$
\dot{\xi}=\left[\begin{array}{c}
\dot{x} \\
\dot{q}
\end{array}\right]=\left[\begin{array}{c}
A x+B \gamma(x, q) \\
0
\end{array}\right], \quad \xi \in \mathcal{C},
$$

where the flow set $\mathcal{C} \subset \mathbb{R}^{n} \times\{0,1\}$ is defined below as the closure of the complement of the jump set. To complete the hybrid system we define the jump map

$$
\xi^{+}:=\left[\begin{array}{l}
x^{+} \\
q^{+}
\end{array}\right]=\left[\begin{array}{c}
x \\
1-q
\end{array}\right], \quad \xi \in \mathcal{D}_{0} \cup \mathcal{D}_{1} .
$$

The jump set $\mathcal{D}=\mathcal{D}_{0} \cup \mathcal{D}_{1}$, responsible for the controller selection $u_{s}$ and $u_{a}$ in (24) by toggling $q$, is defined as

$$
\begin{aligned}
& \mathcal{D}_{0}:=L_{\leq}^{\sigma-h}\left(V_{s}\right) \cup L_{\geq}^{\alpha+h}\left(V_{s a}\right) \times\{1\}, \\
& \mathcal{D}_{1}:=L_{\leq}^{\alpha}\left(V_{s a}\right) \backslash L_{<}^{\sigma}\left(V_{s}\right) \times\{0\},
\end{aligned}
$$

and thus the flow set $\mathcal{C}$ is given by $\mathcal{C}=\overline{\Xi \backslash \mathcal{D}}$ where $\Xi=$ $\mathbb{R}^{n} \times\{0,1\}$. The $x$-component of the jump sets $\mathcal{D}_{0}$ and $\mathcal{D}_{1}$ are defined based on the level sets visualized in Figure 2 and are visualized in Figure 3.

The definition of $\mathcal{D}_{0}$ ensures that if the sublevel set $L_{\leq}^{\sigma-h}\left(V_{s}\right)$ of the Lyapunov function $V_{s}$ is reached, the stabilizing controller $u_{s}$ is used. Since $L_{\leq}^{\sigma-h}\left(V_{s}\right)$ is forward invariant with respect to $u_{s}$ and $L_{<}^{\sigma-h}\left(V_{s}\right)$ does not contain $x_{a}$ according to (23b), the control law $u_{s}$ guarantees asymptotic stability of the origin without leaving $L_{\leq}^{\sigma-h}\left(V_{s}\right)$.

Additionally, the component $L_{>}^{\alpha+h}\left(V_{s a}\right) \times\{1\}$ ensures that the avoidance controller $u_{a v}$ only has local impact. In particular, if we start with an initial value $\xi_{0}=\left(x_{0}^{T}, 1\right)^{T}$
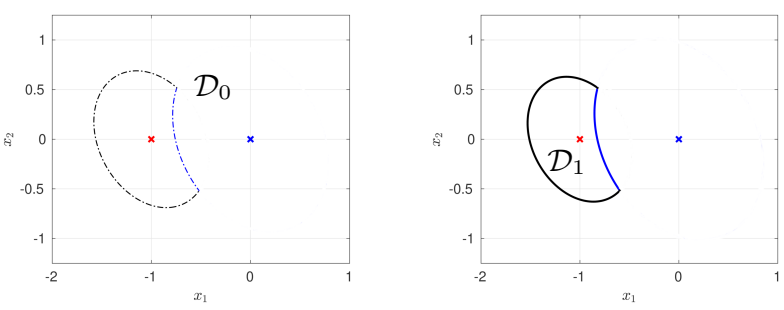

Fig. 3. Visualization of the $x$-component of the jump sets $\mathcal{D}_{0}$ and $\mathcal{D}_{1}$ corresponding to the setting in Figure 2. With slight abuse of notation the $x$-component without $q$-component is denoted as $\mathcal{D}_{0}$ and $\mathcal{D}_{1}$ in the figure.

where the $x$-component $x_{0} \notin L_{\leq}^{\alpha+h}\left(V_{s a}\right)$, the definition of $\mathcal{D}_{0}$ together with the toggling mechanism in (26) ensure that the controller immediately jumps to the stabilizing controller $u_{s}$. As argued in Lemma 1, if we start inside the sublevel set $L_{\leq}^{\alpha+h}\left(V_{s a}\right)$ with the avoidance controller $u_{a}$, then the $x$ component cannot leave $L_{\leq}^{\alpha+h}\left(V_{s a}\right)$ as long as $u_{a}$ is used as an input (namely with $q=1$ ).

The set $\mathcal{D}_{1}$ is defined in such a way that if the sublevel set of the Chetaev function $L_{\leq}^{\delta}\left(C_{d}\right)$ is entered with the stabilizing controller $u_{s}$ and $x$ is not in the forward invariant sublevel set $L_{<}^{\sigma}\left(V_{s}\right)$ of the Lyapunov function $V_{s}$, then the avoidance controller is activated. The margin $h>0$ of the hysteresis parameter is introduced to guarantee robustness of the control law and to avoid Zeno behavior.

Remark 1: Similar to [3, Lemma 2] we point out that the closed-loop dynamics (24)-(27) satisfies the hybrid basic conditions [6, Assumption 6.5] and all maximal solutions are complete.

With these definitions we are able to show the following closed-loop properties of the hybrid system (24)-(27).

Theorem 1: (Obstacle avoidance) For $n \in \mathbb{N}, n \geq 2$, the hybrid system (24)-(27) satisfies the following avoidance properties:

(a) For any initial value $\xi_{0} \in \Xi \backslash\left(\left\{x_{a}\right\} \times\{0,1\}\right)$, the solution $\xi\left(\cdot, \cdot ; \xi_{0}, \gamma\right)$ satisfies

$$
\xi\left(t, j ; \xi_{0}, \gamma\right) \notin\left\{x_{a}\right\} \times\{0,1\} \quad \forall(t, j) \in \operatorname{dom}(\xi) .
$$

(b) For any initial value $\xi_{0} \in \mathbb{R}^{n} \backslash\left(L_{\leq}^{\delta}\left(C_{d}\right) \backslash L_{<}^{\sigma}\left(V_{s}\right)\right) \times$ $\{0\}$, the $x$-component of the solution $\xi\left(\cdot, \cdot ; \xi_{0}, \gamma\right)$ satisfies

$$
x\left(t, j ; \xi_{0}, \gamma\right) \notin L_{\leq}^{\delta}\left(C_{d}\right) \backslash L_{<}^{\sigma}\left(V_{s}\right) \quad \forall(t, j) \in \operatorname{dom}(\xi) .
$$

Proof: The statements (a) and (b) follow immediately from Lemma 1, the properties of the control laws $u_{s}, u_{s a}$, and $u_{d}$, and the definitions of the jump set $\mathcal{D}$.

Item (a): If $\xi\left(t, j ; \xi_{0}, \gamma\right) \in L_{<}^{\sigma-h}\left(V_{s}\right) \times\{0,1\}$ for $(t, j) \in$ $\overline{\operatorname{dom}(\xi)}$ then the definition of $\mathcal{D}_{0}$ and $u_{s}$ ensures that $\xi\left(T, J ; \xi_{0}, \gamma\right) \in L_{<}^{\sigma-h}\left(V_{s}\right) \times\{0,1\}$ for all $(T, J) \in \operatorname{dom}(\xi)$, $(T, J) \geq(t, j)$. If $\xi \in L_{<}^{\delta}\left(C_{d}\right) \times\{0,1\}$, either Lemma 1 ensures that the set $\mathcal{S} \times\{0,1\}$ is reached by the solution without going through $\left\{x_{a}\right\} \times\{0,1\}$ if $\gamma(\xi)=u_{a}(x)$, or the forward invariance of $L_{<}^{\sigma}\left(V_{s}\right)$ ensures that the solution $\xi\left(\cdot, \cdot ; \xi_{0}, \gamma\right)$ does not go through $\left\{x_{a}\right\} \times\{0,1\}$ if $\gamma(\xi)=u_{s}(x)$. Again, by Lemma 1 , it follows that once $\xi\left(\cdot, \cdot ; \xi_{0}, u_{a}\right)$ enters the 
set $\mathcal{S} \times\{0,1\}$ the solution needs to stay in $\mathcal{S} \times\{0,1\}$. The solution can only leave $\mathcal{S} \times\{0,1\}$ if $\gamma(\xi)$ switches to $u_{s}$ and then $x_{a}$ is avoided due to assumption (23c). These considerations also cover the case $\xi \in L_{\geq}^{\delta}\left(C_{d}\right) \times\{0,1\}$.

Item (b): The assumption on the initial condition $\xi_{0}$ ensures that if $x_{0} \in L_{<}^{\alpha}\left(V_{s a}\right) \cap L_{<}^{\sigma}\left(V_{s}\right)$ then $q_{0}=0$ and thus $\gamma(\xi)=$ $u_{s}(x)$ ensures that $x\left(t, j ; \xi_{0}, \gamma\right) \notin L_{\leq}^{\alpha}\left(V_{s a}\right) \backslash L_{<}^{\sigma}\left(V_{s}\right)$ for all $(t, j) \in \operatorname{dom}(\xi)$. If $x_{0} \notin L_{<}^{\delta}\left(C_{d}\right)$, Lemma 1 together with the definition of $\mathcal{D}_{1}$ show the assertion.

The following result establishes (almost) global asymptotic stability for the case $n=2$. Its extension to higher dimensions will be subject of future investigations. The result provides almost global properties since only the point $x_{a}$ needs to be excluded from the stability analysis.

Theorem 2: (Almost global asymptotic stability) For $n=$ 2, the hybrid system (24)-(27) has the following stability property. For any initial value $\xi_{0} \in \mathbb{R}^{n} \backslash\left\{x_{a}\right\} \times\{0,1\}$, the hybrid solution satisfies

$$
\xi\left(t, j ; \xi_{0}, \gamma\right) \rightarrow(0,0)^{T}
$$

for $(t, j) \rightarrow \infty$ and $(t, j) \in \operatorname{dom}(\xi)$, namely the origin is almost globally asymptotically stable.

Proof: First note that local stability follows from the fact that $\mathcal{D}_{0}$ contains a neighborhood of the origin and after a jump to $q=0$ the closed-loop dynamics is linear continuoustime and exponentially stable. It is thus sufficient to show that every solution $\xi\left(\cdot, \cdot ; \xi_{0}, \gamma\right), \xi_{0} \notin\left\{x_{a}\right\} \times\{0,1\}$, converges to the origin to establish asymptotic stability.

The $x$-component of every solution starting outside the set $L_{\leq}^{\alpha}\left(V_{s a}\right) \cup L_{\leq}^{\sigma-h}\left(V_{s}\right)$ reaches the set $L_{\leq}^{\alpha}\left(V_{s a}\right) \cup L_{\leq}^{\sigma-h}\left(V_{s}\right)$ in finite time due the control law $u_{s}$. Combined with the properties of the control law $u_{a}$ derived in Lemma 1, the $x$-component of every solution starting in $x_{0} \in \mathbb{R}^{n} \backslash\left\{x_{a}\right\}$ reaches the set $\mathcal{S} \cup L_{<}^{\sigma-h}\left(V_{s}\right)$ in finite time. Finally, Lemma 3 combined with condition (23b) ensures that the $x$-component of every solution starting in $x_{0} \in \mathbb{R}^{n} \backslash\left\{x_{a}\right\}$ reaches the set $L_{<}^{\sigma-h}\left(V_{s}\right)$ in finite time from where asymptotic convergence follows from the definition of $u_{s}$.

Observe that Theorem 1 combined with Theorem 2 provides a solution to Problem 1 in the planar case $n=2$. In higher dimensions asymptotic stability cannot be guaranteed with our current methods but will be the focus of future research. Surprisingly, for $n \geq 3$ odd, global asymptotic stability cannot hold due to Corollary 1 . Thus, a fundamentally different approach seems to be necessary if possible at all. For $n \geq 4$ even, the statement in Theorem 2 might be true, but we cannot prove that every $x$-component of a solution reaches the set $L_{\leq}^{\sigma-h}\left(V_{s}\right)$ in finite time since we rely on the Poincaré-Bendixson Theorem which is only applicable in the planar setting. Promising directions are given by the recent results in [5].

\section{NUMERICAL EXAMPLE}

\section{A. A planar underactuated linear system}

As a numerical example, we consider the controllable underactuated planar system

$$
\left[\begin{array}{c}
\dot{x}_{1} \\
\dot{x}_{2}
\end{array}\right]=\left[\begin{array}{rr}
0 & 1 \\
-1 & 1
\end{array}\right]\left[\begin{array}{l}
x_{1} \\
x_{2}
\end{array}\right]+\left[\begin{array}{l}
0 \\
1
\end{array}\right] u
$$

The subspace of induced equilibria is given by $\mathcal{E}=$ $\operatorname{span}\left((1,0)^{T}\right)$ and we select the unsafe point $x_{a}=$ $(-1,0)^{T} \in \mathcal{E}$. The feedback gain matrices $K_{s}$ and $K_{s a}$ are obtained through pole placement with $\lambda_{1}=\lambda_{2}=-1$ and $K_{d}$ is obtained through pole placement with $\lambda_{1}=\lambda_{2}=1$.

The Lyapunov functions $V_{s}$ and $V_{s a}$ and the Chetaev function are calculated through the Lyapunov equations (10) for $Q_{s}=Q_{s a}=Q_{d}=I$, where $I \in \mathbb{R}^{2 \times 2}$ denotes the identity matrix. The parameters defining the level sets are selected as $\delta=0.1, \alpha=0.6, \sigma=0.7$, and the hysteresis parameter is set to $h=0.1$. The parameters satisfy the conditions (23), which can be visually verified in Figure 4 . Figure 4 shows

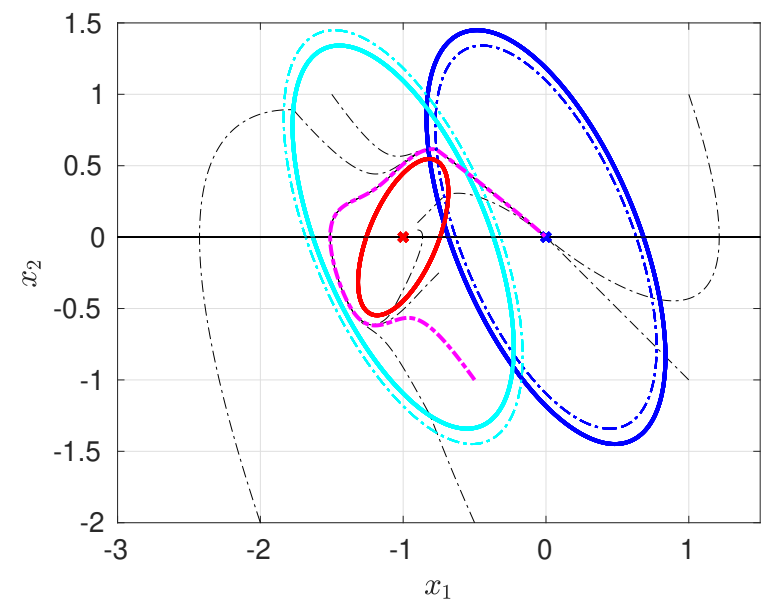

Fig. 4. Visualization of the $x$-component of the solution of the hybrid system (24)-(27) for different initial values. All solutions converge to the origin while avoiding the unsafe point $x_{a}$. The decrease/increase of functions $V_{s}$ and $C_{d}$ as well as the input $\gamma$ with respect to the solution in magenta are shown in Figure 5.

the closed-loop solutions for several initial values $\xi_{0}=$ $\left(x_{0}^{T}, q_{0}\right)^{T}$ using the hybrid control law $\gamma$ defined in (24). As expected all solutions converge to the origin and avoid the unsafe point $x_{a}$. Solutions starting outside the level set $x_{0} \notin$ $L_{\leq}^{\delta}\left(C_{d}\right)$ additionally never enter the set $L_{\leq}^{\delta}\left(C_{d}\right) \backslash L_{<}^{\sigma}\left(V_{s}\right)$. In Figure $5, C_{d}\left(x\left(t ; x_{0}, \gamma(\xi)\right), V_{s}\left(x\left(t ; x_{0}, \gamma(\xi)\right)\right.\right.$ and $\gamma(\xi)$ for the initial value $x_{0}=-(0.5,1)^{T}$ and $q_{0}=1$ are shown. The controller $u_{a}$ ensures that $C_{d}\left(x\left(t ; x_{0}, \gamma(\xi)\right) \geq \delta\right.$ for all $t \in[0,8]$. A monotonic decrease of the Lyapunov function is only guaranteed after $V_{s}\left(\left(x\left(t ; x_{0}, \gamma(\xi)\right) \leq \sigma-h\right.\right.$ and $u_{a}$ is switched to $u_{s}$. A discontinuity in $\gamma(\xi)$ can be observed when the controller is switched. The red lines indicate the level sets $L_{=}^{\sigma-h}\left(V_{s}\right), L_{=}^{\alpha}\left(V_{s a}\right)$ and $L_{=}^{\delta}\left(C_{d}\right)$, respectively. 

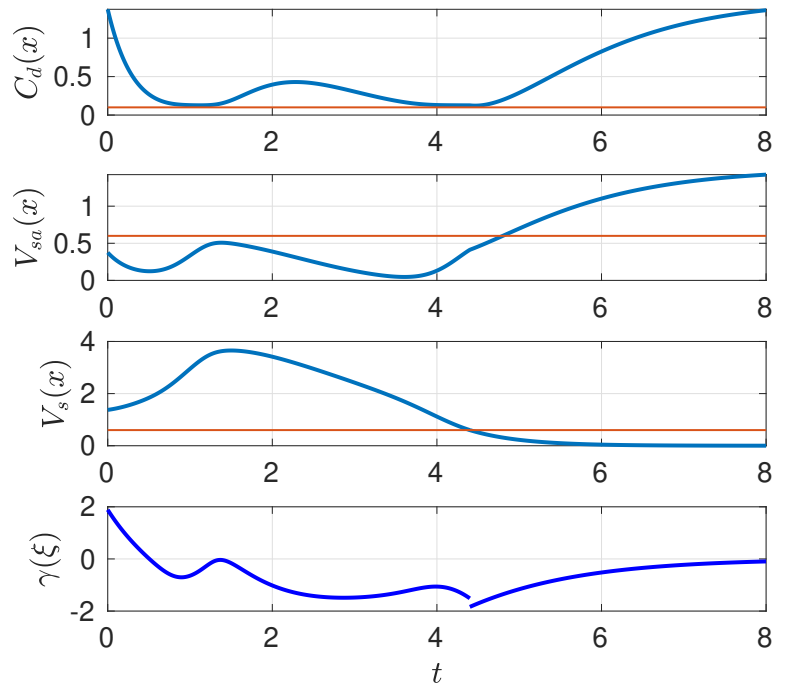

Fig. 5. Decrease/Increase of the functions $V_{s}, V_{s a}$ and $C_{d}$ as well as the input $\gamma$ with respect to the $x$-component of the solution of the hybrid system (24)-(27) for the initial value $\xi_{0}=(-0.5,-1,1)^{T}$. The $x$-component converges to the origin and satisfies $C_{d}\left(x\left(t ; x_{0}, \gamma(\xi)\right) \geq \delta\right.$ for all $t \in$ $[0,8]$.

\section{B. A four dimensional system}

As a second example we consider the controllable four dimensional system

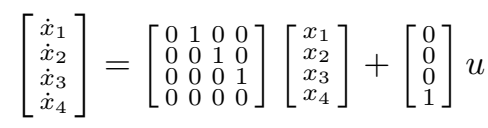

with the subspace of induced equilibria $\mathcal{E}=$ $\operatorname{span}\left((1,0,0,0)^{T}\right)$. For the numerical results shown in Figure 6 , the unsafe point $x_{a}=(2,0,0,0)^{T} \in \mathcal{E}$ and the initial value $x_{0}=(2.1,0.1,0.1,0.1)^{T}, q_{0}=1^{T}$ were
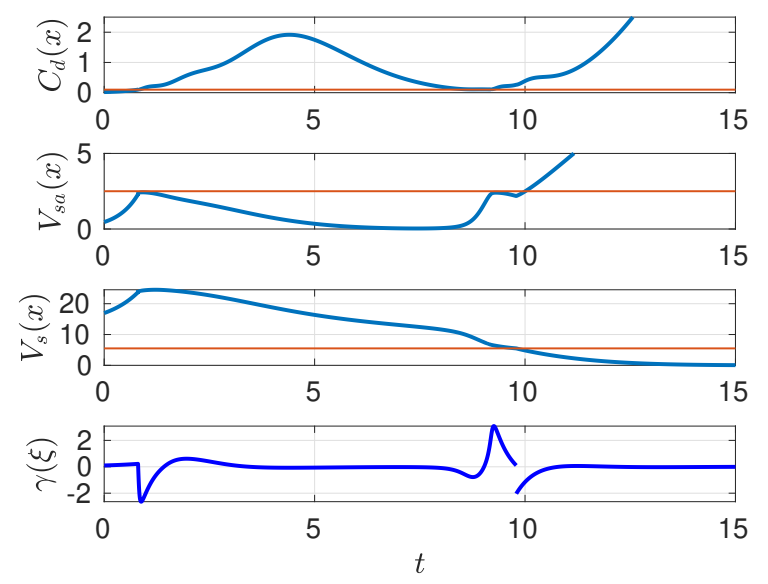

Fig. 6. Decrease/Increase of the functions $V_{s}, V_{s a}$ and $C_{d}$ as well as the input $\gamma$ with respect to the $x$-component of the solution of the hybrid system (24)-(27) for the initial value $x_{0}=(2.1,0.1,0.1,0.1)^{T}, q_{0}=1$ for the linear system (29). Even though not guaranteed by the theoretical results, the closed-loop dynamics show the same avoidance and stability properties as in the two dimensional setting 2 . used. The feedback gain matrices $K_{s}, K_{s a}, K_{d}$ and the corresponding Lyapunov/Chetaev functions were again obtained through pole placement using the eigenvalues -1 and 1 , and the identity matrix in the Lyapunov equations (10). The level sets of the Lyapunov and the Chetaev functions are defined through the parameters $\delta=0.1$, $\alpha=2.5, \sigma=5.5$, and $h=0.1$. The solution visualized in Figure 6 shows the same closed-loop behavior as the two dimensional setting in Figure 5 even though asymptotic stability is not guaranteed through Theorem 2 .

\section{CONCLUSIONS}

In this paper we extend earlier work in [3] and [4] addressing the problem of robust controller design mechanisms for underactuated linear systems. In particular, we study hybrid switching control laws which simultaneously guarantee the avoidance of a neighborhood around an unsafe point $x_{a}$ and convergence of solutions to the origin. In contrast to the work in [3] and [4], in this paper we consider unsafe points in the one dimensional subspace $x_{a} \in\left\{y \in \mathbb{R}^{n}: \exists \nu \in\right.$ $\mathbb{R}, A y+B \nu=0\}$. While we provide a complete solution for planar linear systems, convergence results of the proposed controller in higher dimension $n>2$ are still open and will be the subject of future investigations.

\section{REFERENCES}

[1] A. G. Akritas, E. K. Akritas, and G. I. Malaschonok. Various proofs of Sylvester's (determinant) identity. Mathematics and Computers in Simulation, 42(4-6):585-593, 1996.

[2] P. Braun, L. Grüne, and C. M. Kellett. Complete instability of differential inclusions using Lyapunov methods. In Proc. of the 57th IEEE Conference on Decision and Control, pages 718-724, 2018.

[3] P. Braun, C. M. Kellett, and L. Zaccarian. Explicit construction of robust avoidance controllers for linear systems. IEEE Transactions on Automatic Control, 2018. (submitted), Preprint: https://hal.archivesouvertes.fr/hal-01893027/.

[4] P. Braun., C. M. Kellett, and L. Zaccarian. Unsafe point avoidance in linear state feedback. In Proc. of 57th IEEE Conference on Decision and Control, pages 2372-2377, 2018.

[5] F. Forni and R. Sepulchre. Differential dissipativity theory for dominance analysis. IEEE Transactions on Automatic Control, 2018. DOI: 10.1109/TAC.2018.2867920.

[6] R. Goebel, R. G. Sanfelice, and A. R. Teel. Hybrid Dynamical Systems: modeling, stability, and robustness. Princeton University Press, 2012.

[7] O. Khatib. Real-time obstacle avoidance for manipulators and mobile robots. In Proc. of the IEEE International Conference on Robotics and Automation, pages 500-505, March 1985.

[8] O. Khatib. Real-Time Obstacle Avoidance for Manipulators and Mobile Robots, pages 396-404. Springer New York, 1990.

[9] D. Liberzon. Switching in Systems and Control. Birkhäuser, Boston, MA, 2003.

[10] J. D. Meiss. Differential dynamical systems. SIAM, 2007.

[11] A. N. Michel, L. Hou, and D. Liu. Stability of dynamical systems. Springer, 2008.

[12] C. Prieur. Uniting local and global controllers with robustness to vanishing noise. Mathematics of Controls, Signals and Systems, 14:143-172, 2001.

[13] C. Prieur and A. R. Teel. Uniting local and global output feedback controllers. IEEE Transactions on Automatic Control, 56(7):16361649,2011

[14] J. J. Sylvester. On the relation between the minor determinants of linearly equivalent quadratic functions. The London, Edinburgh, and Dublin Philosophical Magazine and Journal of Science, 1(4):295-305, 1851.

[15] P. Wieland and F. Allgöwer. Constructive safety using control barrier functions. IFAC Proceedings Volumes, 40(12):462-467, 2007. 